\title{
Resource Allocation Optimization Model of Collaborative Logistics Network Based on Bilevel Programming
}

\author{
Xiao-feng Xu, Wei-hong Chang, and Jing Liu \\ College of Economics and Management, China University of Petroleum, Qingdao 266580, China \\ Correspondence should be addressed to Xiao-feng Xu; xuxiaofeng@upc.edu.cn
}

Received 14 July 2016; Accepted 23 January 2017; Published 22 February 2017

Academic Editor: Tomàs Margalef

Copyright (C) 2017 Xiao-feng Xu et al. This is an open access article distributed under the Creative Commons Attribution License, which permits unrestricted use, distribution, and reproduction in any medium, provided the original work is properly cited.

\begin{abstract}
Collaborative logistics network resource allocation can effectively meet the needs of customers. It can realize the overall benefit maximization of the logistics network and ensure that collaborative logistics network runs orderly at the time of creating value. Therefore, this article is based on the relationship of collaborative logistics network supplier, the transit warehouse, and sellers, and we consider the uncertainty of time to establish a bilevel programming model with random constraints and propose a genetic simulated annealing hybrid intelligent algorithm to solve it. Numerical example shows that the method has stronger robustness and convergence; it can achieve collaborative logistics network resource allocation rationalization and optimization.
\end{abstract}

\section{Introduction}

Collaborative logistics network is a supply and demand network, which consists of supply collaboration nodes such as raw materials and equipment and logistics function collaboration nodes such as transportation and warehousing and even road and relationship between nodes [1]. The allocation of resource is to meet the supply and demand network. The aim is to collaborate logistics network enterprise internal and external resources and combine the supply chain system effectively which is composed of producers, manufacturers, and customers to realize the lowest cost and the best quality service [2]. So, reasonable resource allocation process is the basis to realize the orderly operation of the whole logistics network. However, collaborative logistics network has suppliers, transit warehouses, vendors, and other forms of logistics node and has some characteristics such as being dynamic, open, and complex. It leads to some uncertainty factors in the process of allocating logistics resources that may have an effect on the running time and operating cost. Therefore, this research about resource allocation of collaborative logistics network is how to select nodes in the numerous suppliers and transit warehouse under the condition of considering the influence of uncertainty factors to realize the optimal logistics system.
The current research on collaborative logistics network resource scheduling is not enough in depth and it is mainly concentrated in the delivery path optimization and distribution address selection as well as the relationship between collaboration node and so forth. In terms of path selection, Yu et al. [3] established a scheduling model with time as constraint and the shortest route as object and used artificial intelligence algorithms to solve the problem. Najera [4] used evolutionary algorithm to study transportation routing problem in consideration of capacity of vehicle. Mir and Abolghasemi [5] took vehicle transportation situations of encoring or not into account and proposed the optimal route according to the customer location changes. Chen et al. [6] made full use of geographic information system technology to select path under the constraint of the least distribution sites. In terms of optimal location selection, Cheng et al. [7] study the impact of the rapid transit on the capacity of current urban transportation system, and a twomode network capacity model, including the travel modes of automobile and transit, is developed based on the wellknown road network capacity model. Turskis and Zavadskas [8] used the fuzzy multiple criteria decision to describe fuzziness of site selection of distribution center. Nozick and Turnquist [9] established a location optimization decision model from the aspects of cost and customer responsiveness. 
Yang and Zhou [10] built the location selection model with equilibrium constraints considering facility competition on the basis of the equilibrium theory and then applied genetic algorithm and projection algorithm to solve the model. For the relationship between collaboration points, Qiu and Wang [11] develop a robust optimization model for designing a three-echelon supply chain network that consists of manufacturers, distribution centers, and retailers under both demand uncertainty and supply disruptions. Liu et al. [12] considered three-layer logistics service supply chain and studied the order task allocation model between collaboration nodes.

Integrating the documents, we can find that the current research focused on the microscopic aspects, such as path optimization and site selection and used artificial intelligence algorithm to solve the model. But the ideal model cannot fully describe the collaborative allocation of resources due to the network's complexity and some uncertainty factors in the process of resource allocation. Collaborative logistics network resource allocation process involves three levels, which are suppliers, transit warehouse, and vendors, so the bilevel programming model can be used to explain the relationship between every two levels of nods. At the same time, the stochastic constrained programming can protect model against uncertain influence, and it has been applied to the system dynamics, structural dynamics, and financial and other fields [13-15]. Therefore, this article will set up the three-level nodes relationship among the suppliers, transit warehouses, and retailers and establish a bilevel programming model with chance constrained to control uncertainty factors, so as to make the whole logistics network system optimal under meeting the nodes' profit of both sides.

Generally speaking, the bilevel programming is NP-hard problem, Ben-Ayed and Blair (1988) have proved that [16] this problem does not have a polynomial algorithm, so the solution of it is very complicated. Like genetic algorithm (GA) and Simulated Annealing Algorithm (Simulated Annealing, SA), Neural Network Algorithm (NNA), and so forth, some intelligent algorithms were used to solve the bilevel programming problem. Li et al. [17] put forward a genetic algorithm that can effectively solve bilevel programming problem. In this method, they used constraint to transform bilevel problem into a single-level one and designed the binary coding to solve the multiplier, and numerical experiment shows that the given algorithm can find the global optimal solution in the least time. Niwa et al. [18] proposed a bilevel programming problem that the upper class has only one decision-maker while the lower one has multiple decisionmakers and a distribution of the genetic algorithm. Li and Wang [19] studied a special kind of linear quadratic bilevel programming problems, and genetic algorithm was proposed after being converted into equivalent problem.

Genetic algorithm by the ideas of the fittest in nature chooses an optimal individual for the solution of the model by selection, crossover, and mutation genetic operation. Although it has some characteristics such as simple operation, strong operability, and problem space independence, it is easy to exhibit slow convergence speed and get only local optimal value [20]. Simulated annealing algorithm is the result of logistics annealing principle of solid matter, starting from an initial temperature to find the optimal solution in the solution space with the reduction of the temperature parameters. Although it can get the global optimal solution, it evolved slowly and has strong dependence on parameters [21]. So there are advantages and disadvantages, respectively, of the two algorithms, hybrid genetic simulated annealing algorithms can compensate deficiency for each other, they not only can search the related areas of optimal solution in the global but also can find the optimal solution in the region of the optimal solution, and there have been some scholars who have done the related research. Wang and Zheng [22] proposed a hybrid heuristic algorithm, the algorithm mixed the genetic algorithm and simulated annealing method and the sampling process of simulated annealing method instead of mutation operators of genetic algorithm, and this algorithm improves the local search ability of genetic algorithm. Wang et al. [23] assume that the drivers all make route choices based on Stochastic User Equilibrium (SUE) principle. Two methods, that is, the sensitivity analysis-based method and genetic algorithm (GA), are detailedly formulated to solve the bilevel reserve capacity problem. Kong et al. [24] establish a bilevel programming model of land use structure indexes variables and use GASA to solve the problem, and land and road area ratio should be improved by numerical example results. So in this paper designing the combination of genetic and simulated annealing hybrid algorithm, to solve the bilevel programming problem, is feasible.

\section{Considering Uncertainty Resource Deployment of Bilevel Programming Model}

2.1. Bilevel Programming Model. Bilevel programming (BP) [25] model was presented by Bracken and McGill in 1973, the lower decision-maker makes decision in the first place, the upper policymakers must predict the possible reaction of the lower ones, and then the lower one reacts according to the decision of the upper one to optimize the objective function of the individual. The general model is

$$
\begin{array}{ll}
\min _{x \in X} & F(x, y) \\
\text { s.t. } & G(x, y) \leq 0, \\
\min _{y \in Y} & f(x, y) \\
\text { s.t. } & g(x, y) \leq 0 .
\end{array}
$$

Among them, $x \in R^{n_{1}}$ and $y \in R^{n_{2}}$. The upper variable is $x \in R^{n_{1}}$, and the lower variable is $y \in R^{n_{2}}$. Also, the functions $F: R^{n_{1}} \times R^{n_{2}} \rightarrow R$ and $f: R^{n_{1}} \times R^{n_{2}} \rightarrow R$ are the upper and the lower objective function, respectively, and vector-valued functions $G: R^{n_{1}} \times R^{n_{2}} \rightarrow R^{m_{1}}$ and $g: R^{n_{1}} \times R^{n_{2}} \rightarrow R^{m_{2}}$ are, respectively, the upper and the lower constraint conditions.

\subsection{Chance Constrained Programming Model. Chance Con-} strained Programming [26] is a method of stochastic programming put forward by Charnes and Cooper in 1959. It allows decision value within a certain range of fluctuations 
considering the possibility that decision-making process may not meet the constraint conditions, but the probability of the constraint set-up must not be less than a certain confidence level $\alpha$ which is small enough. The general model is

$$
\begin{array}{ll}
\min & Z(x)=\sum_{j=1}^{n} c_{j} x_{j} \\
\text { s.t. } & \operatorname{Pr}\left[\sum_{j=1}^{n} a_{1 j} x_{j} \geq b_{1}, \ldots, \sum_{j=1}^{n} a_{m j} x_{j} \geq b_{m}\right] \geq \alpha \\
& x_{j} \geq 0 \quad j=1,2, \ldots, n .
\end{array}
$$

Among them, $\operatorname{Pr}[$ ] is the probability of the event set-up, $\alpha$ is the confidence probability for the condition, $b_{m}$ are random variables that obey a certain distribution, and $a$ and $b$ obey $N\left(\mu_{1}, \sigma_{1}^{2}\right)$ and $N\left(\mu_{2}, \sigma_{2}^{2}\right)$, respectively.

\subsection{The Model of Collaborative Logistics Network Resource} Allocation considering Uncertainty. This paper researches on the resource allocation optimization decision of multiple suppliers and multiple warehouse transfer nodes and retailers. The raw materials price and transportation fee that each suppliers charge are different, and it is a key issue for the retailers to select the optimization suppliers and carry resources more effectively to their warehouses transfer node. At the same time, the retailers compare the transit fees cost of each warehouse, choosing the lowest one or more warehouses to allocation according to their own requirements, which need to focus on every step of the whole logistics network. This kind of relationship can be explained by bilevel programming model. Described in this paper, the upper programming's goal is how the suppliers realize the profit maximization of their own on the condition of meeting the warehouse requirements; the lower level programming describes the lowest cost during the process of warehouse transit center and retailer.

In order to understand better, we can use mathematical language to describe the problem as follows.

Assume that the logistics network $N$ consists of suppliers $\left\{S_{i} \mid i=1,2, \ldots, n_{i}\right\}$, transit warehouses $\left\{M_{j} \mid j=\right.$ $\left.1,2, \ldots, n_{j}\right\}$, retailers $\left\{P_{k} \mid k=1,2, \ldots, n_{k}\right\}$, and links between each node $\left\{R \mid R \in R_{S_{i} M_{j}} \cup R_{M_{j} P_{k}}\right\}$, including logistics nodes $\{O \mid O \in S \cup M \cup P\}$. When retailer $P_{k}$ sends the demand quantity $D_{l}^{P_{k}}$ of resource $\left\{l \mid l=1,2, \ldots, n_{l}\right\}$, the resource will be transported to $P_{k}$ from warehouses $M_{j}$. $X_{M_{j} P_{k}}^{l}$ stands for the quantity of products $l$ that are distributed to the retailers $P_{k}$ from the warehouses $M_{j} ; X_{S_{i} M_{j}}^{l}$ stands for the quantity of raw materials that are distributed to the warehouses $M_{j}$ from the suppliers $S_{i} \cdot\left\{E(R) \mid E(R) \in E\left(R_{S_{i} M_{j}}\right) \cup E\left(R_{M_{j} P_{k}}\right)\right\}$ stand for the distance between all logistics nodes. Due to the different location of logistics nodes, the product fees of transportation, storage, packaging, and processing for each unit are also different. Therefore, $\{C(R) \mid C(R) \in$ $\left.C\left(R_{S_{i} M_{j}}^{l}\right) \cup C\left(R_{M_{j} P_{k}}^{l}\right)\right\}$ stand for the cost of each unit distance between different logistics nodes to transport resources $l$; $C\left(f_{O}^{l}\right)$ and $C\left(g_{O}^{l}\right)$ stand for the unit cost of storage and processing (including labor) for all logistics nodes. Suppliers as the disclosing party, charge transit warehouses $C\left(X_{S_{i} M_{j}}^{l}\right)$ for the resources $l$. Considering that all logistic nodes have a certain amount of time requirement to order processing and distribution of resources, the times of delivery requirement from warehouses to retailers and supplies to warehouses, respectively, are $T_{M_{j} P_{k}}^{l}$ and $T_{S_{i} M_{j}}^{l}$, the times of order processing and production processing, respectively, are $T_{M_{j} P_{k}}^{l}{ }^{\prime}, T_{S_{i} M_{j}}^{l}{ }^{\prime}$, $T_{M_{j} P_{k}}^{l}$ ", and $T_{M_{j} P_{k}}^{l}$; transport vehicle maximum loading capacities, respectively, are $G_{M_{j} R_{k}}$ and $G_{S_{i} M_{j}}$.

In the process of research, the assumptions made are as follows for the purpose of simplified model:

(1) All land transportation is between all logistics nodes.

(2) The logistics node routes and distance have to be known and are fixed.

(3) Vehicle average speed is $V$ during the process of transportation between various logistics nodes.

\subsubsection{The Upper Programming considering the Relationship between Suppliers and Warehouse Transit Centers}

$$
\begin{aligned}
& \max F_{1}=\sum_{j=1}^{n} \sum_{k=1}^{n} \sum_{l=1}^{n} \sum_{i=1}^{n}\left(C\left(X_{S_{i} M_{j}}^{l}\right)-C\left(R_{S_{i} M_{j}}^{l}\right) E\left(R_{S_{i} M_{j}}\right)-C\left(f_{S_{i}}^{l}\right)-C\left(g_{S_{i}}^{l}\right)\right) X_{S_{i} M_{j}}^{l} u_{i} \\
& \sum_{i=1}^{n} u_{i} \geq 1 \\
& G_{S_{i} M_{j}} \geq X_{S_{i} M_{j}}^{l}{ }^{\prime}\left[T_{S_{i} M_{j}}^{l}+T_{S_{i} M_{j}}^{l}+\frac{E\left(R_{S_{i} M_{j}}\right)}{V} \leq T_{S_{i} M_{j}}^{l}\right]=\alpha \\
& u_{i} X_{S_{i} M_{j}}^{l}=X_{M_{j} P_{k}}^{l}
\end{aligned}
$$




$$
\begin{aligned}
& X_{S_{i} M_{j}}^{l} \geq 0 \quad i=1,2, \ldots, n, j=1,2, \ldots, n \\
& u_{i} \in\{0,1\} .
\end{aligned}
$$

Among them, (3) is from the point of view of the suppliers, which is pursuing of the profit maximization; (4) ensures warehouse transit centers at least choose one supplier; (5) means the amount of resources that the suppliers provide cannot exceed its maximum of vehicle loading capacity; (6) stands for the probability that order processing time, processing time, and shipping time of supplies cannot exceed the longest time of warehouse transit centers which is $\alpha$; (7) means the amount of resources that warehouse transit centers provide is equal to what all suppliers provide; (8) resource demand is a positive number; (9) is supplier's 0 - 1 variable constraints, which means that select supplier $i$ has a value of 1 or a value of 0 .

\subsubsection{The Lower Programming considering the Relationships} between Warehouse Transit Centers and Retailers

$$
\begin{aligned}
& \min F_{2} \\
& =\sum_{j=1}^{n} \sum_{k=1}^{n} \sum_{l=1}^{n} \sum_{j=1}^{n}\left(C\left(R_{M_{j} P_{k}}^{l}\right) E\left(R_{M_{j} P_{k}}\right)+C\left(f_{M_{j}}^{l}\right)+C\left(g_{M_{j}}^{l}\right)\right) \\
& \quad \cdot X_{M_{j} P_{k}}^{l} u_{j} \\
& \sum_{j=1}^{n} u_{j} \geq 1 \\
& \sum_{j=1}^{n} \sum_{k=1}^{n} \sum_{j=1}^{n} u_{j} X_{M_{j} P_{k}}^{l} \geq D_{l}^{P_{k}} \\
& G_{M_{j} R_{k}} \geq X_{M_{j} P_{k}}^{l} \\
& \operatorname{Pr}\left[T_{M_{j} P_{k}}^{l}+T_{M_{j} P_{k}}^{l}+\frac{E\left(R_{M_{j} P_{k}}\right)}{V} \leq T_{M_{j} P_{k}}^{l}\right]=\beta \\
& X_{M_{j} P_{k}}^{l} \geq 0 \quad j=1,2, \ldots, n, k=1,2, \ldots, n \\
& u_{j} \in\{0,1\} .
\end{aligned}
$$

Among them, expression (10) stands for the warehouse transit center and distributors as a whole, pursuing the minimizing cost including transportation and storage and producing cost; (11) ensures that retailers choose at least one supply warehouse transit center; (12) means the total resources that product warehouse transit centers provide should meet the demand of retailers $P_{k}$; (13) means the amount of resources that warehouse transit centers provide cannot exceed its maximum of vehicle loading capacity; (14) stands for the probability that the order processing time, processing time, and shipping time of warehouse transit centers cannot exceed the longest time of retailers which is $\beta$; (15) means resource demand is a positive number; (16) is warehouse transit centers' $0-1$ variable constraints, which means that chosen warehouse $j$ has a value of 1 or value of 0 .

\section{The Solution to the Resource Allocation Bilevel Programming Model considering Uncertainty}

3.1. Random Planning Constraints Transformation. As for the uncertain factors during transportation and order processing, we need to transform them into deterministic constraints. The solution method of stochastic programming constraint probably has two kinds: one is transforming the stochastic programming into a deterministic mathematical programming through certain changes and then using the existing method that solves the deterministic mathematical programming to solve; the other method is to use the intelligent algorithm such as neural network based on the idea of approximation function.

Due to solving bilevel programming after stochastic programming constraints transformation, we take the first method to research. The order processing times $T_{M_{j} P_{k}}^{l}$ and $T_{S_{i} M_{j}}^{l}{ }^{\prime}$, respectively, obey the normal distributions $T_{M_{j} P_{k}}^{l} \sim N\left(\mu\left(T_{M_{j} P_{k}}^{l}{ }^{\prime}\right), \sigma\left(T_{M_{j} P_{k}}^{l}{ }^{\prime}{ }^{2}\right)\right.$ and $T_{S_{i} M_{j}}^{l}{ }^{\prime} \sim N\left(\mu\left(T_{S_{i} M_{j}}^{l}{ }^{\prime}\right)\right.$, $\sigma\left(T_{S_{i} M_{j}}^{l}{ }^{\prime}{ }^{2}\right) ; T_{M_{j} P_{k}}^{l}{ }^{\prime \prime}$ and $T_{S_{i} M_{j}}^{l}$, respectively, obey the normal distributions $T_{M_{j} P_{k}}^{l} " \sim N\left(\mu\left(T_{M_{j} P_{k}}^{l}{ }^{\prime \prime}\right), \sigma\left(T_{M_{j} P_{k}}^{l}{ }^{\prime \prime}\right)^{2}\right)$ and $T_{S_{i} M_{j}}^{l}{ }^{\prime \prime} \sim N\left(\mu\left(T_{S_{i} M_{j}}^{l}{ }^{\prime \prime}\right), \sigma\left(T_{S_{i} M_{j}}^{l}{ }^{\prime \prime}\right)^{2}\right) ; E\left(R_{M_{j} P_{k}}\right) / V$ and $E\left(R_{S_{i} M_{j}}\right) / V$, respectively, obey the normal distributions $E\left(R_{M_{j} P_{k}}\right) / V \sim N\left(\mu\left(E\left(R_{M_{j} P_{k}}\right) / V\right), \sigma\left(E\left(R_{M_{j} P_{k}}\right) / V\right)^{2}\right)$ and $E\left(R_{S_{i} M_{j}}\right) / V \sim N\left(\mu\left(E\left(R_{S_{i} M_{j}}\right) / V\right), \sigma\left(E\left(R_{S_{i} M_{j}}\right) / V\right)^{2}\right)$.

So the stochastic programming constraint (7)

$$
\operatorname{Pr}\left[T_{S_{i} M_{j}}^{l}{ }^{\prime}+T_{S_{i} M_{j}}^{l}{ }^{\prime \prime}+\frac{E\left(R_{S_{i} M_{j}}\right)}{V} \leq T_{S_{i} M_{j}}^{l}\right]=\alpha
$$

can be transformed into

$$
\begin{array}{r}
\mu\left(T_{S_{i} M_{j}}^{l}{ }^{\prime}\right)+\phi^{-1}(\alpha) \sigma\left(T_{S_{i} M_{j}}^{l}{ }^{\prime}\right)+\mu\left(T_{S_{i} M_{j}}^{l}{ }^{\prime \prime}\right) \\
+\phi^{-1}(\alpha) \sigma\left(T_{S_{i} M_{j}}^{l}{ }^{\prime \prime}\right)+\mu\left(\frac{E\left(R_{S_{i} M_{j}}\right)}{V}\right) \\
+\phi^{-1}(\alpha) \sigma\left(\frac{E\left(R_{S_{i} M_{j}}\right)}{V}\right) \leq T_{S_{i} M_{j}}^{l}
\end{array}
$$




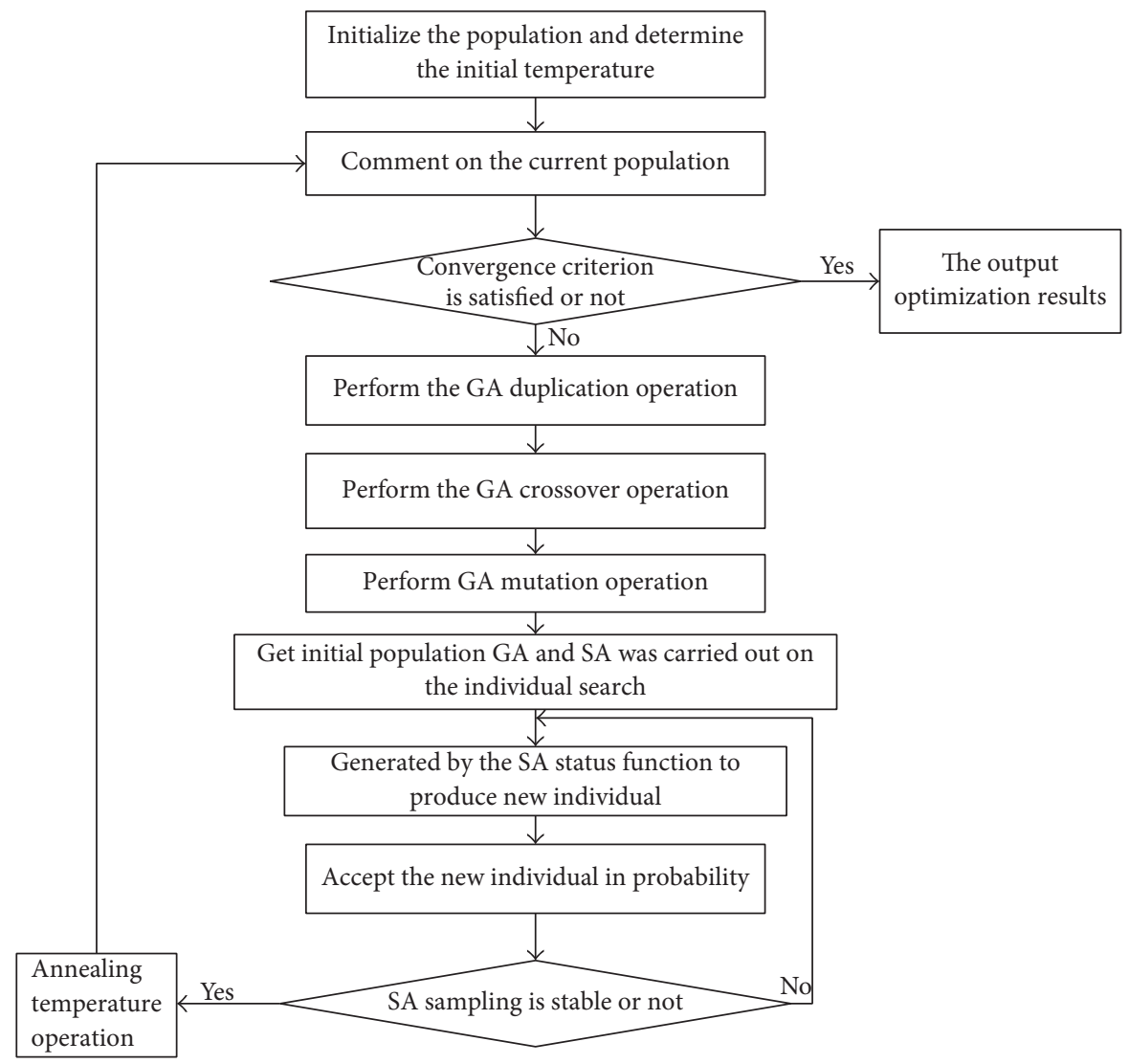

FIGURE 1: The solving steps of genetic simulated annealing algorithm.

and the stochastic programming constraint (14)

$$
\operatorname{Pr}\left[T_{M_{j} P_{k}}^{l}{ }^{\prime}+T_{M_{j} P_{k}}^{l}{ }^{\prime \prime}+\frac{E\left(R_{M_{j} P_{k}}\right)}{V} \leq T_{M_{j} P_{k}}^{l}\right]=\beta
$$

can be transformed into

$$
\begin{gathered}
\mu\left(T_{M_{j} P_{k}}^{l}{ }^{\prime}\right)+\phi^{-1}(\beta) \sigma\left(T_{M_{j} P_{k}}^{l}{ }^{\prime}\right)+\mu\left(T_{M_{j} P_{k}}^{l}{ }^{\prime \prime}\right) \\
+\phi^{-1}(\beta) \sigma\left(T_{M_{j} P_{k}}^{l}{ }^{\prime \prime}\right)+\mu\left(\frac{E\left(R_{M_{j} P_{k}}\right)}{V}\right) \\
+\phi^{-1}(\beta) \sigma\left(\frac{E\left(R_{M_{j} P_{k}}\right)}{V}\right) \leq T_{M_{j} P_{k}}^{l} .
\end{gathered}
$$

3.2. The Solving Thought of Genetic Simulated Annealing Algorithm. The basic idea of genetic simulated annealing algorithm is as follows: firstly encoding the upper planning variables and solving the lower programming to calculate the fitness of each string. And then one can get the best series through copy, crossover, mutation, and simulated annealing. Specific steps are shown as Figure 1.
Step 1. Initialization

(1-1) Set parameters, including the crossover probability of genetic algorithm $P_{c}$, mutation probability $P_{m}$, each generation population of individuals (chromosomes) number $N$, and largest evolution algebra Maxgen. Set the evolution algebra gen $=0$

(1-2) Confirm the number of inner loops $M$ and the initial value of the temperature $T_{o}$ of simulated annealing algorithm; let $T=T_{o}$

(1-3) Determine a reasonable fitness function according to the objective function $F_{1}$ of upper programming, determine the encoding way of the decision variable $u$ of upper programming, and randomly generate initial population $X(1)=\left(\ldots, x_{i}(1), \ldots\right), i=1,2, \ldots, N$; let gen $=1$

Step 2. Take $X$ (gen) into lower programming to be UE distribution calculation, and calculate the fitness of each individual $x_{i}$ (gen) $(i=1,2, \ldots, N)$. If gen = Maxgen, the largest fitness chromosome is the optimal solution of resource allocation; else, turn to Step 3.

Step 3. Copy group $X$ (gen) according to the fitness distribution. 
Step 4. It is crossover operation according to the crossover probability $P_{c}$.

Step 5. Perform mutation operation according to the mutation probability $P_{m}$; let gen $=$ gen +1 , get a new population $X$ (gen), and then calculate the fitness of individuals of $X$ (gen).

Step 6. For $i=1$, conducting the simulated annealing of species $X$ (gen) is as follows:

(6-1) If $i=N$, turn to Step 7; otherwise the cycle counting round $k=1$, and turn to (6-2).

(6-2) Get individual $x_{i}$ (gen) by state function, then decode new individuals, and conduct UE distribution calculation under lower programming to get the objective function value of the upper programming, and calculate the fitness.

(6-3) Accept new individual under Metropolis probability acceptance formula.

(6-4) if $k=M, i=i+1$, turn to (6-1); else, $k=k+1$, turn to $(6-2)$.

Step 7. Annealing temperature: let $T=0.5 T$, and turn to Step 2.

Description is as follows:

(1) The upper decision variables generally take binary encoding; multivariate encoding is as follows:

\begin{tabular}{cr|c|c|c} 
decision variables $u=u_{1}$ & $u_{2}$ & $\ldots$ & $u_{n}$ \\
mapping & $\downarrow$ & $\downarrow$ & $\downarrow$ & $\downarrow$ \\
Chromosome (string) $x=0011$ & 1011 & $\ldots$ & 0110.
\end{tabular}

(2) In (1-3), the relationship between the length of the substring $\beta$ and the precision of decision variables $\pi$ is

$$
\beta \geq \log _{2}\left(\frac{u_{\max }-u_{\min }}{\pi}+1\right)
$$

(3) In a (6-2), SA state function can do random exchange of two different genes positions of chromosome and reverse genes order of different random position of chromosome, such as

$$
\begin{array}{cc|c}
\text { Decision variables } u= & u_{1} & u_{2} \\
\text { Genes Position } & 25 & 37 \\
\text { Chromosome } x= & 01100101 & 10110101 .
\end{array}
$$

Assume that the random variable, respectively, positions are 2 and 3 and 5 and 7 , and the new individuals after exchanging and reversing are

$$
\begin{array}{l|l}
x_{1}=00101101 & 10010111 \\
x_{2}=00011101 & 10010111 .
\end{array}
$$

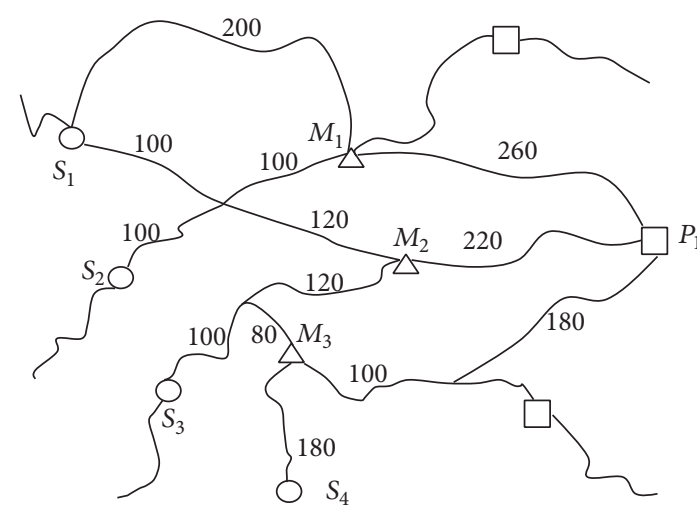

$$
\begin{array}{lll}
\bigcirc \text { Supplies } & \square & \text { Retailers } \\
\triangle \text { Transit node } & \underbrace{40} \begin{array}{l}
\text { Distance between nodes } \\
\text { (unit: } \mathrm{km} \text { ) }
\end{array}
\end{array}
$$

FIGURE 2: Production and sale collaborative logistics network of one sneaker brand.

TABLE 1: The distance between each supplier and transit warehouse.

\begin{tabular}{lccc}
\hline & $M_{1} / \mathrm{km}$ & $M_{2} / \mathrm{km}$ & $M_{3} / \mathrm{km}$ \\
\hline$S_{1}$ & 200 & 240 & $\infty$ \\
$S_{2}$ & 240 & 220 & $\infty$ \\
$S_{3}$ & $\infty$ & 220 & 180 \\
$S_{4}$ & $\infty$ & $\infty$ & 180 \\
\hline
\end{tabular}

\section{The Sample Simulation}

4.1. Sample. Taking the collaborative logistics network production-sales of one sneaker brand as an example, a product retailer put forward a demand of 4000 pieces of goods to the transit warehouse according to the sales plan and product orders. Assume that we can choose goods from four suppliers and three transit warehouses that are available, vehicle average speed is 40 kilometers per hour between various logistics nodes in the process of transportation, the biggest load capacities from suppliers to the transit warehouses and from transit warehouses to retailers are, respectively, 2000 and 3000, and specific arrangement is shown in Figure 2.

The distance between all suppliers to the transit warehouse is shown in Table 1; the suppliers' storage and processing fees for each unit are shown in Table 2. The distance between each transit warehouse to the retailer and the transit warehouses' storage and processing fees for each unit are shown in Table 3.

4.2. Solving Example. The actual, material delivery time that warehouse transit node and retailer require is 10 hours and 12 hours, because of that the supplier and warehouse transit point have some uncertain factors such as the order processing and production, time obeys the normal distribution as Table 4 shows, there are also uncertainty factors on transport which obey the normal distribution table as shown in Table 5, value interval is $\mu \pm 3 \sigma$, namely, the probability that the value points fall in the interval is $99.73 \%$, and the probability 
TABLE 2: The material charge and cost for each unit of storage and transportation of suppliers.

\begin{tabular}{lcccc}
\hline & $\begin{array}{c}\text { Material } \\
\text { charges } \\
(\mathrm{RMB})\end{array}$ & $\begin{array}{c}\text { Storage } \\
\text { charges } \\
(\mathrm{RMB})\end{array}$ & $\begin{array}{c}\text { Processing } \\
\text { charges } \\
(\mathrm{RMB})\end{array}$ & $\begin{array}{c}\text { Transportation } \\
\text { charges } \\
(\mathrm{RMB})\end{array}$ \\
\hline$S_{1}$ & 110 & 0.1 & 0.15 & 0.02 \\
$S_{2}$ & 100 & 0.15 & 0.15 & 0.03 \\
$S_{3}$ & 110 & 0.1 & 0.1 & 0.035 \\
$S_{4}$ & 100 & 0.15 & 0.1 & 0.025 \\
\hline
\end{tabular}

TABLE 3: The distance between retailer and transit warehouses and transit warehouses' cost for unit of storage and transportation.

\begin{tabular}{ccccc}
\hline & $\begin{array}{c}\text { Distance } \\
(\mathrm{km})\end{array}$ & $\begin{array}{c}\text { Storage } \\
\text { charges } \\
(\mathrm{RMB})\end{array}$ & $\begin{array}{c}\text { Processing } \\
\text { charges } \\
(\mathrm{RMB})\end{array}$ & $\begin{array}{c}\text { Transportation } \\
\text { charges } \\
(\mathrm{RMB})\end{array}$ \\
\hline$M_{1}$ & 260 & 0.2 & 0.25 & 0.02 \\
$M_{2}$ & 220 & 0.3 & 0.2 & 0.025 \\
$M_{3}$ & 280 & 0.2 & 0.15 & 0.015 \\
\hline
\end{tabular}

TABLE 4: The uncertain index value of the supplier and warehouse transfer point's order processing and production (unit: hours).

\begin{tabular}{lcc}
\hline & $\begin{array}{c}\text { Order } \\
\text { processing time }\end{array}$ & $\begin{array}{c}\text { Production and } \\
\text { processing time }\end{array}$ \\
\hline $\begin{array}{l}\text { Supplier } \\
\begin{array}{l}\text { Storage and } \\
\text { transit point }\end{array}\end{array}$ & $N(0.1,5)$ & $N(1,5)$ \\
\hline
\end{tabular}

TABLE 5: The uncertain index value of transport time range of suppliers to transit warehouse and warehouse transfer to seller (unit: hours).

\begin{tabular}{lccc}
\hline & $M_{1}$ & $M_{2}$ & $M_{3}$ \\
\hline$S_{1}$ & $N(5,5)$ & $N(6,5)$ & $\infty$ \\
$S_{2}$ & $N(6,5)$ & $N(5.5,5)$ & $\infty$ \\
$S_{3}$ & $\infty$ & $N(5.5,5)$ & $N(4.5,5)$ \\
$S_{4}$ & $\infty$ & $\infty$ & $N(4.5,5)$ \\
Seller & $N(6.5,5)$ & $N(5.5,5)$ & $N(7,5)$ \\
\hline
\end{tabular}

that ensures finishing the logistics tasks in the time of the warehouse transit node and retailer is $90 \%$, which means $\alpha$ and $\beta$ both are $90 \%$.

The parameters of the algorithm are as follows: the population size is 50 , the crossover probability is 0.6 , the probability of mutation is 0.1 , the cooling coefficient is 0.95 , and the initial temperature is 100 . The result converges to the optimal solution after 45 generations by using Matlab7.0 for many times while traditional genetic algorithm iteration number is 64, as shown in Figure 3. The optimal solution in the genetic simulated annealing algorithm is shown in Table 5 .

It can be seen as shown in Table 6 that supplier $S_{1}$ deployed 1000 unit materials to transit warehouse $M_{1}$, supplier $S_{4}$, respectively, deployed 1999.99 and 1000.01 unit materials to $M_{2}$ and $M_{3}$, on the basis of meeting the expected

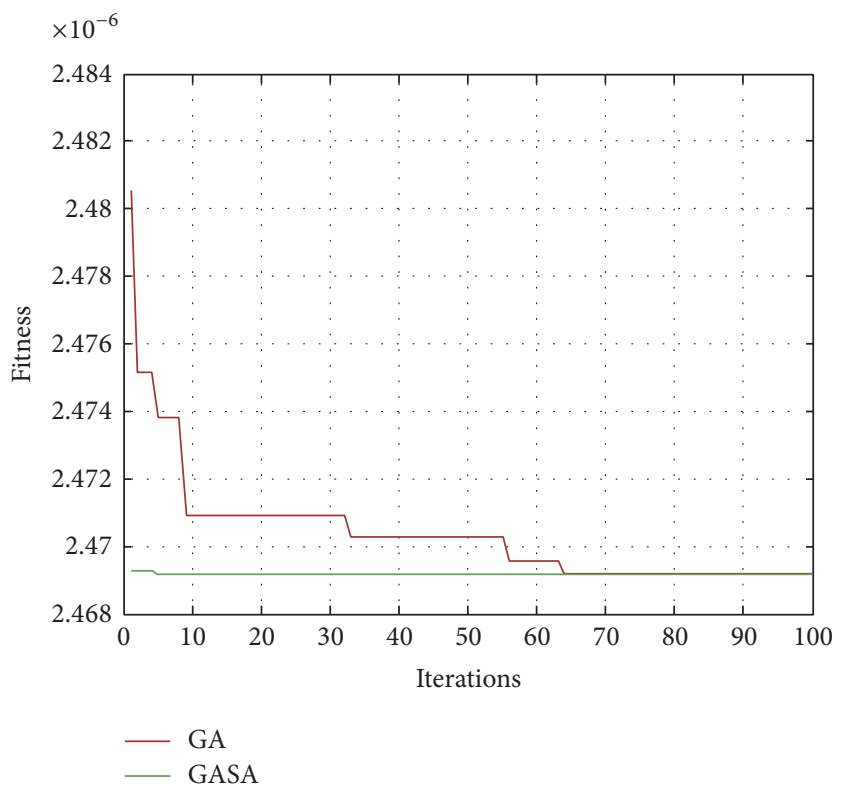

FIGURE 3: The convergence comparison chart of genetic simulated annealing algorithm and genetic algorithm.

TABLE 6: The resources allocation for each supplier to the warehouse transfer point.

\begin{tabular}{lccc}
\hline & $M_{1}$ & $M_{2}$ & $M_{3}$ \\
\hline$S_{1}$ & 1000 & & \\
$S_{2}$ & & & \\
$S_{3}$ & & & \\
$S_{4}$ & & 1999.99 & 1000.01 \\
\hline
\end{tabular}

delivery time, and it makes the supplier's profit maximum 405000 Yuan while retailer's cost is minimum about 19300 Yuan while, on the basis of traditional genetic algorithm, the maximum profit of suppliers is 404990 Yuan. The result indicates that the solution can maximize the interests of both sides, which verify the operability and optimization of the model.

\section{Conclusion}

Collaborative logistics network is a virtual organization, which is led by manufacturing/service companies or independent third-party logistics enterprise. It is a supply and demand network made up of supply collaboration nodes, function of logistics nodes, and the link road and relationship between nodes; the core task is overall plan and deployment access of the network node resources to realize the overall interests and meet the customer service. Therefore, this paper establishes a supply-sales network on the basis of collaborative logistics network resource allocation model, which includes collaborative logistics network suppliers, warehouse transit nodes, retailers, and their node link. From retailer's demand as a starting point, considering the shipping time and quantity and distribution cost factors, we establish a bilevel programming model with uncertainty factors. On this basis, 
we use the genetic simulated annealing algorithm to analyze and solve the model, which can get the optimal scheme that can not only meet suppliers benefit maximization but also make cost minimum to retailers. The analysis results show the feasibility and validity of the model, which can provide the optimal resource allocation decisions and plans.

\section{Competing Interests}

The authors declare that there is no conflict of interests regarding the publication of this paper.

\section{Acknowledgments}

This research was supported by the National Natural Science Foundation of China (Grant no. 71501188), the Natural Science Foundation of Shandong Province, China (Grant no. ZR2015GQ006), and the Fundamental Research Funds for the Central Universities, China (Grant no. 15CX04100B).

\section{References}

[1] X.-F. Xu, J.-L. Zhao, and J.-K. Song, "Uncertain control optimization of resource planning for collaborative logistics network about complex manufacturing," System Engineering Theory \& Practice, vol. 32, no. 4, pp. 799-806, 2012.

[2] L. H. Shan and Z. Y. Zhang, "Analysis on logistics network system operation mode based on dissipative structure," Logistics Science-Technology, vol. 12, no. 28, pp. 86-89, 2014.

[3] B. Yu, Z. Z. Yang, and B. Z. Yao, "A hybrid algorithm for vehicle routing problem with time windows," Expert Systems with Applications, vol. 38, no. 1, pp. 435-441, 2011.

[4] A. G. Najera, "The vehicle routing problem with backhauls: a multi-objective evolutionary approach," Evolutionary Computation in Combinatorial Optimization, vol. 7245 , no. 42, pp. 255266, 2012

[5] H. S. A. Mir and N. Abolghasemi, "Aparticle swarm optimization algorithm for open vehicle routing problem," Expert Systems with Applications, vol. 38, no. 9, pp. 11547-11551, 2011.

[6] Y. Chen, R. Z. Ruan, and M. C. Yan, "Algorithm of path optimization for physical distribution based on GIS," Geospatial Information, vol. 2, no. 10, pp. 104-182, 2012.

[7] L. Cheng, M. Du, X. Jiang, and H. Rakha, "Modeling and estimating the capacity of urban transportation network with rapid transit," Transport, vol. 29, no. 2, pp. 165-174, 2014.

[8] Z. Turskis and E. K. Zavadskas, "A new fuzzy additive ratio assessment method (ARAS-F). Case study: the analysis of fuzzy multiple criteria in order to select the logistic centers location," Transport, vol. 25, no. 4, pp. 423-432, 2010.

[9] L. K. Nozick and M. A. Turnquist, "Inventory, transportation, service quality and the location of distribution centers," European Journal of Operational Research, vol.129, no. 2, pp. 362-371, 2001.

[10] Y. X. Yang and G. G. Zhou, "Study on location model of facility competition for closed-loop supply chain network with random demands," Control and Decision, vol. 10, no. 26, pp. 1553-1561, 2011.

[11] R. Qiu and Y. Wang, "Supply chain network design under demand uncertainty and supply disruptions: a distributionally robust optimization approach," Scientific Programming, vol. 2016, Article ID 3848520, 15 pages, 2016.

[12] W. H. Liu, S. Y. Qu, and S. Y. Zhong, "Order allocation in three-echelon logistics service supply chain under stochastic environments," Computer Integrated Manufacturing Systems, vol. 2, no. 18, pp. 381-388, 2012.

[13] Q. Zeng and X.-Z. Xu, "Assessing the reliability of a multistate logistics network under the transportation cost constraint," Discrete Dynamics in Nature and Society, vol. 2016, Article ID 2628950, 8 pages, 2016.

[14] P. Guo, G. H. Huang, and Y. P. Li, "An inexact fuzzychance-constrained two-stage mixed-integer linear programming approach for flood diversion planning under multiple uncertainties," Advances in Water Resources, vol. 33, no. 1, pp. 81-91, 2010.

[15] B. Aouni, C. Colapinto, and D. La Torre, "A cardinality constrained stochastic goal programming model with satisfaction functions for venture capital investment decision making," Annals of Operations Research, vol. 205, pp. 77-88, 2013.

[16] X. Zhang, H. Wang, and W. Wang, "Bi-level programming model and algorithms for stochastic network with elastic demand," Transport, vol. 30, no. 1, pp. 117-128, 2015.

[17] H. Li, Y. Jiao, and L. Zhang, "Orthogonal genetic algorithm for solving quadratic bi-level programming problems," Journal of Systems Engineering and Electronics, vol. 21, no. 5, pp. 763-770, 2010.

[18] K. Niwa, T. Hayashida, M. Sakawa, and Y. Yang, "Computational methods for decentralized two level 0-1 programming problems through distributed genetic algorithms," AIP Conference Proceedings, vol. 1285, no. 1, pp. 1-13, 2010.

[19] H. C. Li and Y. P. Wang, "A genetic algorithm for solving linearquadratic bi-level programming problems," in New Trends and Applications of Computer-Aided Material and Engineering, pp. 626-630, Trans Tech Publications, 2012.

[20] S. Yu, Q. Yang, J. Tao et al., "Product modular design incorporating life cycle issues-Group Genetic Algorithm(GGA) based method," Journal of Cleaner Production, vol. 19, no. 9-10, pp. 1016-1032, 2011.

[21] B. Sankararao and C. K. Yoo, "Development of a robust multiobjective simulated annealing algorithm for solving multiobjective optimization problems," Industrial \& Engineering Chemistry Research, vol. 50, no. 11, pp. 6728-6742, 2011.

[22] L. Wang and D.-Z. Zheng, "A modified evolutionary programming for flow shop scheduling," International Journal of Advanced Manufacturing Technology, vol. 22, no. 7, pp. 522-527, 2003.

[23] J. Wang, W. Deng, and J. Zhao, "Road network reserve capacity with stochastic user equilibrium," Transport, vol. 30, no. 1, pp. 103-116, 2015.

[24] Z. Kong, X. Guo, and J. Hou, "Urban land structure planning model based on green transportation system principal," in Proceedings of the 10th International Conference of Chinese Transportation Professionals-Integrated Transportation Systems: Green, Intelligent, Reliable (ICCTP '10), Beijing, China, August 2010.

[25] J. Bracken and J. T. McGill, "Mathematical programs with optimization problems in the constraints," Operations Research, vol. 21, pp. 37-44, 1973.

[26] A. Charnes and W. W. Cooper, "Chance-constrained programming," Management Science, vol. 6, no. 1, pp. 73-79, 1960. 

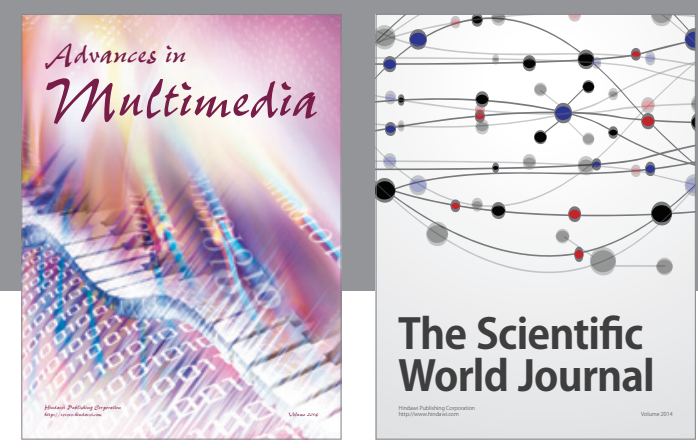

The Scientific World Journal
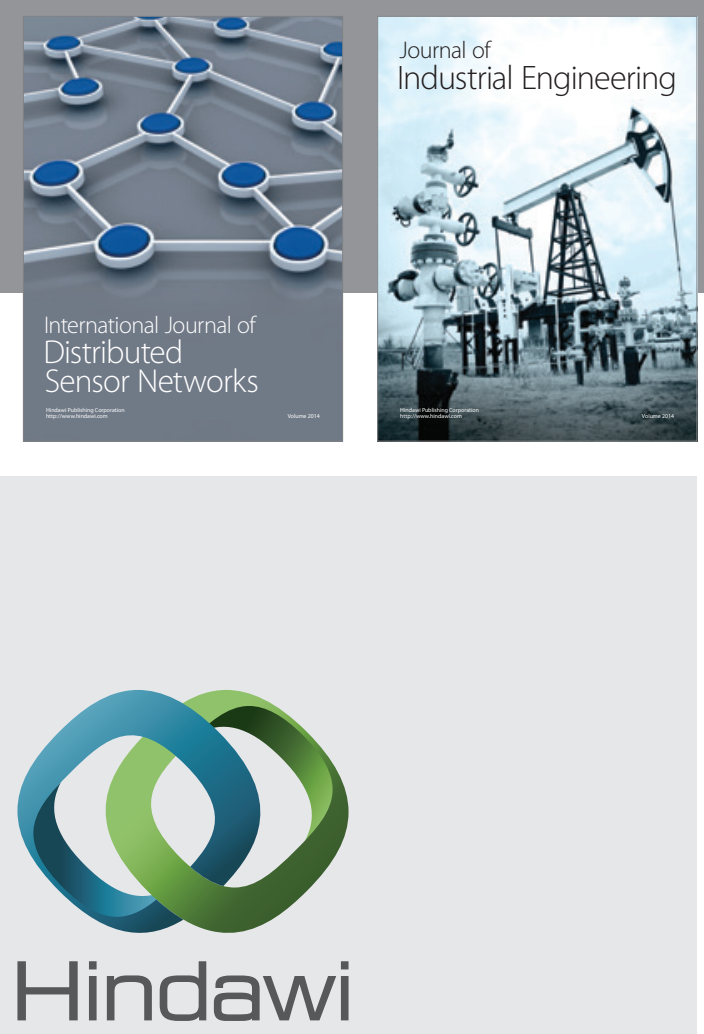

Submit your manuscripts at

https://www.hindawi.com

\section{Computer Networks} and Communications
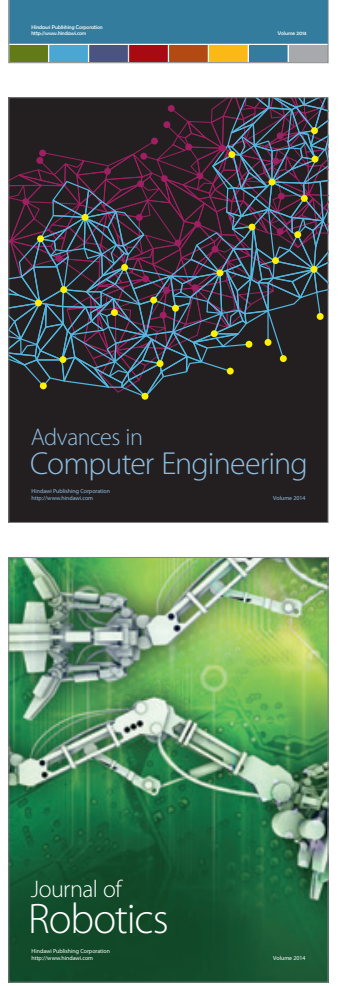
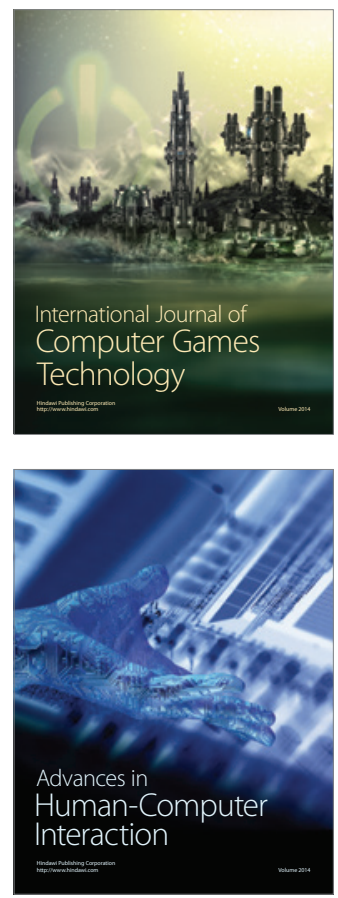
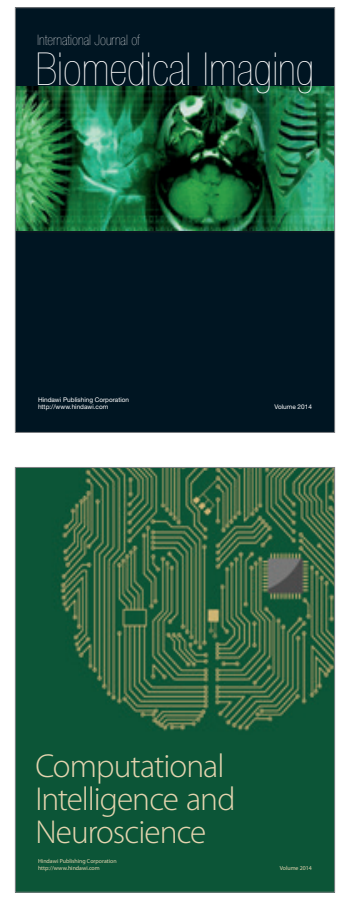
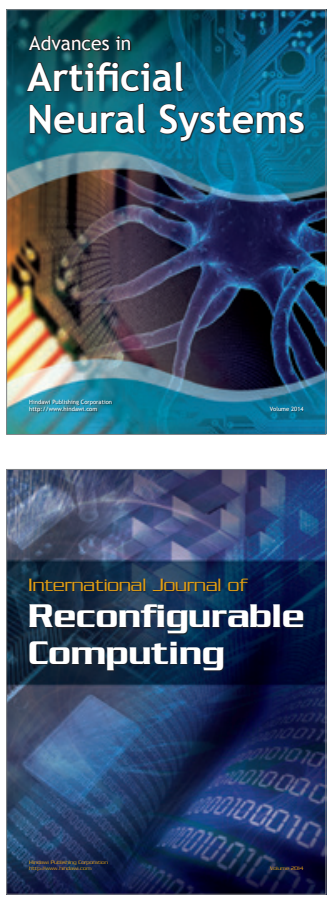
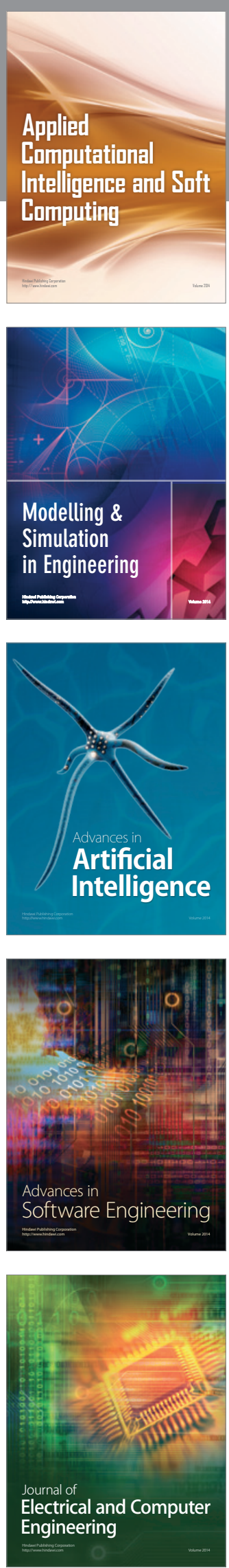